\title{
Study of Fault Diagnosis Method Based On the Hybrid Circuit Simulation
}

\author{
Ting Liu, Xianglian Xu, Tiao Liu, Qiangqiang Yin, Xiaoqian Tang \\ School of automation, WuHan University of Technology, WuHan 430070, China \\ nataly2010@163.com, xuxianglian@163.com, liutiao@whut.edu.cn
}

Keywords: Test; Simulation; Hierarchy; Fault injection; Fault diagnosis

\begin{abstract}
A fault diagnosis method is presented in this paper for poor field testing conditions of large-scale hybrid circuit, and taking the LNE navigation control card which is vital to the operation of regulation and monitoring system in the nuclear power plant, a simulation model of hybrid analog-digital electronic circuits is built with Cadence OrCAD. By extracting the output response characteristics of normal card in the way of hierarchy and setting selection rules of fault mode with the method of software part combination, the wave of fault feature is extracted and thus a fault dictionary is generated, the whole process of fault diagnosis is completed.
\end{abstract}

\section{Introduction}

In the new stage of the steady and efficient development of nuclear power plant, digitization, automation, intelligence has become the new development trend of power plant. All kinds of regulation system and monitor types are various. As the power source of these control systems, the LNE system puts forward more strict requirements to the reliability of the cards which make the system run normally. However, System long-running and human operational errors will lead to hidden faults. Therefore, an example of navigation control card will be taken in this paper, by using the theory of "software is instruments" [1], the method of fault diagnosis for hard fault which has a greater impact is studied with the combination of the circuit simulation technology.

\section{The improvement of diagnostic methods}

The navigation control card has large amount of circuit components and hybrid analog-digital electronic circuits. When the field test condition is poor, it is essential to apply a new kind of diagnostic method. Firstly we should avoid using the method of hardware fault injection, instead of, with the help of simulation to compile fault dictionary; Secondly, applying the method of fault guess, listing the common fault; Again, consideration should also be given to that whether the fault test is comprehensive, so the hierarchical method is applied for fault detection on card; Finally, the detected fault feature signal in comparison with the fault dictionary will provide the results of fault diagnosis. The fault dictionary method based on hybrid simulation The commonly used fault injection method includes physical injection and simulation injection, the simulation injection has the advantage of that, failure is accurately controllable, the method is easy to change, easy to quantify, and low cost, and can avoid the fault injection which may lead to destruction of the device or system [2].

The navigation control card has the hybrid analog-digital circuit, how to make the analog circuit and the digital circuit work together properly is in the area of concern. The Cadence OrCAD software will be applied to realize the co-simulation. The steps are as follows:

The first step, selecting the device in the component database and connect them to complete the input of circuit; The second step, selecting the desired type of simulation, for example, transient analysis; The third step, inputting stimulus to analog circuit and digital circuit respectively, setting the sources as the input of analog circuit and setting the pulse signal as the input of digital circuit; The forth step, assigning simulation task to analog circuit and digital circuit respectively; The fifth step, generating simulation net list of the circuit; The sixth step, executing a simulation task by simulator; The seventh step, generating simulation results in the way of text file or wave file, thus to obtain the 
fault dictionary. The text file can be changed to the wave image which can be easily called and shown in the way of reading by computer.

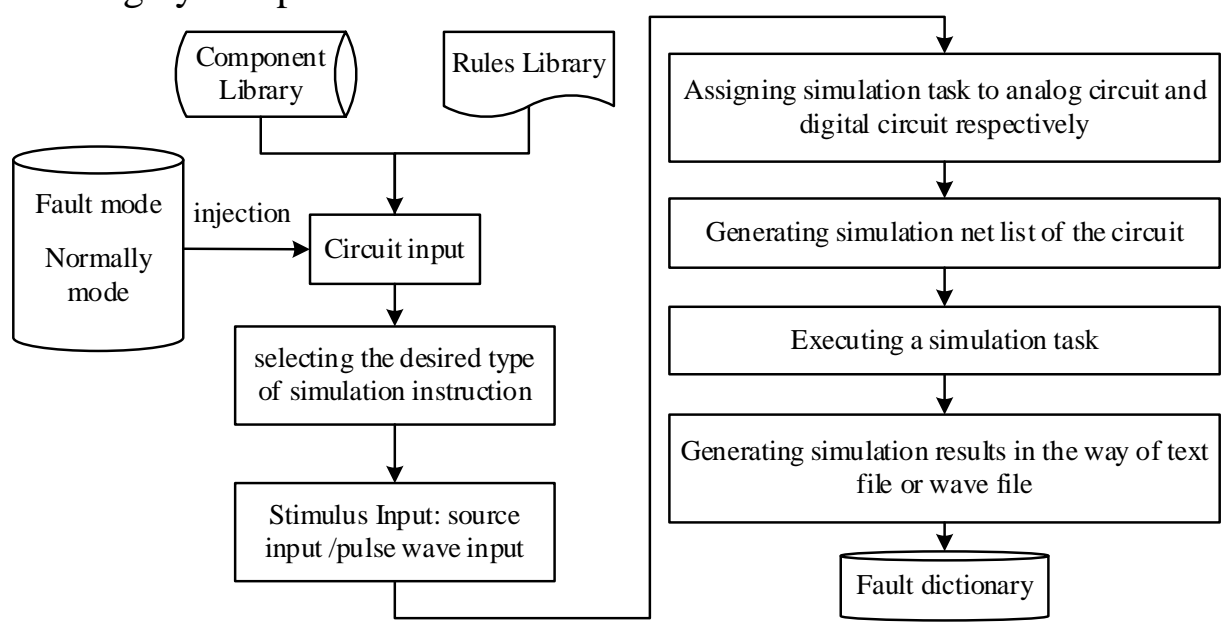

Fig 1 The process of hybrid circuit simulation

The fault detection based on hierarchy analysis In the process of fault detection, it is need a large number of measure times in the way of fault enumeration and cannot locate the fault fast [4]. So the hierarchy analysis method will be applied in this paper, by locating the fault in a hierarchical way which is from card to components, and avoid spending a lot of time traversing the fault.

First of all, according to the circuit board level, the cards are divided into module level, channel circuit level, component level. The navigation control card belongs to the circuit board level, and the module level is a circuit combination which can complete a kind of function, for example, the navigation control card can be divided into DC bus voltage control module, thyristor fault alarm module, SCR trigger module etc. The channel circuit level is a component combination which can complete a kind of function, for example, the DC bus voltage control module can be divided into comparator circuit, filter circuit etc; The component level is the minimum part constituting the circuit board, for example, the comparator circuit can be divided into an operational amplifier, resistors etc. If there are no faults when detecting the module level of navigation control card, then the board level without failure; Or else the specific functional modules is founded, and its corresponding channel circuit is tested; if there are faults in the specific channel circuit, its corresponding components is continue to be tested, and finally the point of failure can be located.

\section{Case study}

The establishment of fault model Because of the complex circuit structure of navigation control card, the following aspects must be considered when creating a simulation model:

(1) The channel circuits without important components can be partly simplified. For example, the photovoltaic coupling components can be replaced by diodes etc.

(2) Network labels are used to adjust the distribution of circuit model, and to reduce redundant circuit. The red mark in the circuit is for the network label, and network labels with the same name are for the same connection node [4].

(3) Transient analysis is selected for simulation command category, and a reasonable simulation step and simulation accuracy must be set to ensure that the circuit operation process can converge and to speed up the computation time. In this paper, the current calculation accuracy is set to $0.01 \mathrm{u}$, while voltage calculation accuracy is $10 \mathrm{u}$, and the relative accuracy of calculation of voltage and current is at 0.05 .

(4) Switch is used, then the switching time can be set to achieve timing control of the power on process of the circuit.

(5) When the probe is used to judge the action of relay, it can be judged by NC, NO terminals between COM end of the relay model (NC is a closed node, and NO is an open one). 
As there is no model library for fault component in Cadence OrCAD, the fault model must be created before the fault is introduced. The method can be used as follows when introducing fault:

(1) Corresponding parameters in the simulation model of components can be directly modified. For impedance devices, by changing the characteristic impedance values of the components can model the fault caused by the parameter.

(2) A new model of the component is series or parallel to the normal simulation model, and then it's packaged as a whole to form a fault simulation model of the component. The newly added component can be resistance, time control switch, or custom fault component

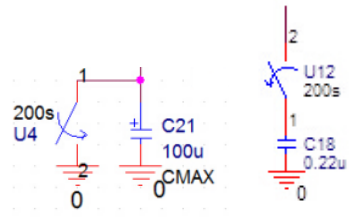

Fig 2 An example of Short circuit model (left) and open circuit model (right)

Generation of fault dictionary The fault dictionary can be generated by extracting fault feature when the fault model is introduced. Due to a combination of multi-fault state is less in the practical application [5], in this paper the dictionary is only for single fault.

Firstly the navigation control card should be divided. Then normal mode motivation is introduced based on functional principle, and the output of the card is measured when it function normally. Secondly, fault is introduced into normal model. The selection of fault mode should be based on the following two tips for rapid diagnosis:

(1) The mode with high fault rate in the running of the navigation control card should be selected. For example, the electrolytic capacitor is easy to age in the long process of charge and discharge, resulting in parameter drift, and even affecting the filter performance [6].

(2) The mode of high degree of influence in the actual operation of the navigation control card after the fault should be selected. For example, high power resistor will generate much heat in the long-term work, resulting in high temperature and the surrounding circuit cannot work normally. In this paper, fault modes and the voltage types to be measured are listed in the following:

Table 1, List of fault mode

\begin{tabular}{|c|c|c|c|}
\hline $\begin{array}{l}\text { Component } \\
\text { Name }\end{array}$ & Fault Mode & $\begin{array}{l}\text { Component } \\
\text { Type }\end{array}$ & Voltage types to be measured \\
\hline \multirow{2}{*}{ C1 } & C1 Short circuit & \multirow{2}{*}{$\begin{array}{l}\text { Electrolytic } \\
\text { capacitor }\end{array}$} & \multirow{2}{*}{$\begin{array}{l}\text { The output response voltage of card, the } \\
\text { response voltage of electrolytic capacitor }\end{array}$} \\
\hline & C1 Open circuit & & \\
\hline \multirow{2}{*}{ R46 } & R46 Short circuit & \multirow{2}{*}{$\begin{array}{l}\text { High power } \\
\text { resistor }\end{array}$} & \multirow{2}{*}{$\begin{array}{l}\text { The output response voltage of card, the } \\
\text { response voltage of high power resistor }\end{array}$} \\
\hline & R46 Open circuit & & \\
\hline \multirow{2}{*}{ RG } & RG Input short circuit & \multirow{2}{*}{$\begin{array}{l}\text { Three terminal } \\
\text { voltage }\end{array}$} & \multirow{2}{*}{$\begin{array}{l}\text { The output response voltage of card, the } \\
\text { response voltage of three terminal voltage }\end{array}$} \\
\hline & RG Input open circuit & & \\
\hline
\end{tabular}

The process of fault diagnosis When the navigation control card goes wrong, firstly the normal power supply is introduced, then $48 \mathrm{~V}$ DC voltage and 230V DC voltage are injected according to the time sequence to simulate DC bus voltage control function of the card. If it functions well, then the output voltage level should be high when the RT_COM1 and RT_NC1 port are measured by probe, and the output voltage level should be high between the RD_COM1 and RD_NC1 port When 75V DC voltage is injected, it shows that thyristor control function is normal if the output voltage level does not change. The output of relay is as the following figure, and other measures are by the same. 


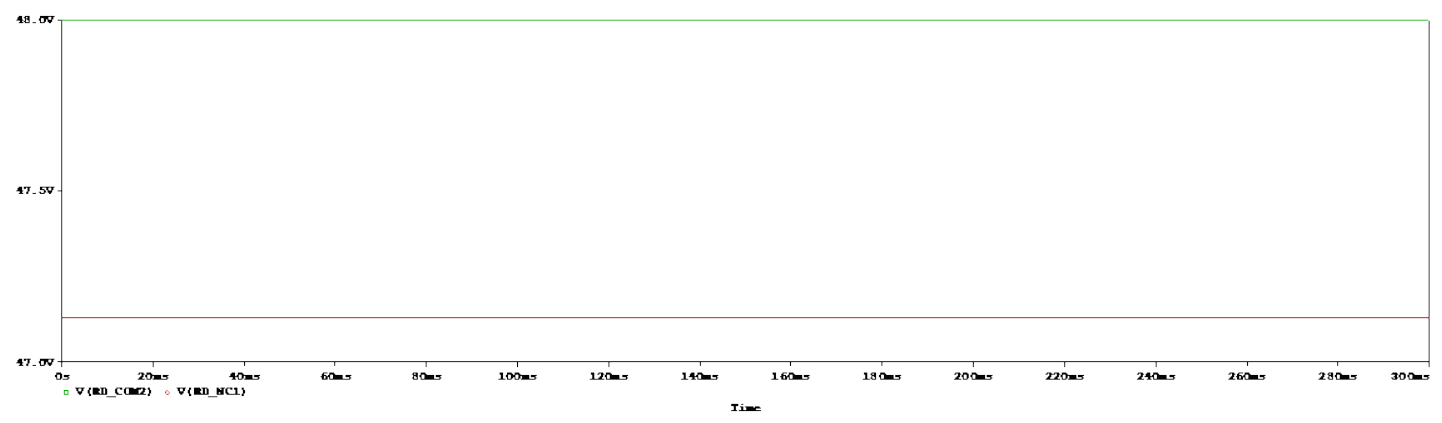

Fig 3 The output response under normal mode

The channel circuit should be tested when the output response wave is not matching during the test. For example, the output response wave of function modules related to voltage control like comparator circuit, voltage stabilizing circuit and so on will be extracted when the DC bus voltage control function goes wrong. When the input of comparator circuit is Abnormal, then it's key to extract the output waveform of the voltage regulator circuit, and targeted test and waveform extraction should be conducted among the components included in the voltage regulator circuit. For example, for the $\mathrm{C} 1$ capacity in the voltage regulator circuit, it can be diagnosed as closed circuit fault if the output voltage drop to zero volts compared to the normal output is between $10 \mathrm{pV}$ to $15 \mathrm{pV}$ by measuring the output voltage waveform. The according waveform is shown as the following figure.

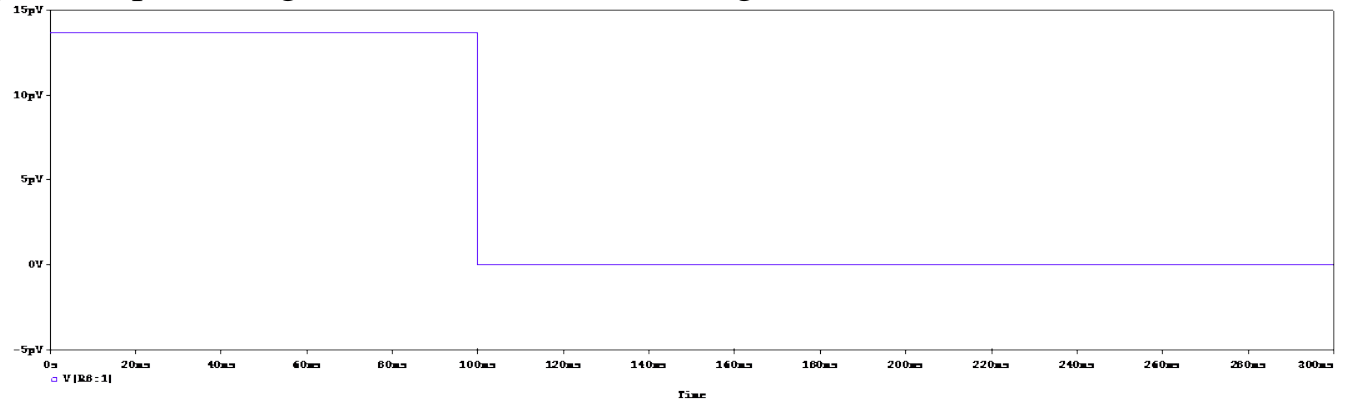

Fig 4 The output response of the component port when C1 capacity is closed

\section{Summary}

Hybrid circuit simulation technology is applied to fault diagnosis in this paper, by functional division of cards, a simulation model of hybrid analog-digital electronic circuits is built with Cadence OrCAD. By extracting the output response characteristics of normal card in the way of hierarchy and setting selection rules of fault mode with the method of software part combination, the wave of fault feature is extracted and thus a fault dictionary is generated. Taking the LNE navigation control card which is vital to the operation of regulation and monitoring system in the nuclear power plant, a simulation model is built in this paper, and the process of fault diagnosis is described in detail. This diagnosis method is also can be applied to other kind of large scale digital analog hybrid circuit diagnosis. With this method the fault can be located fast in the case of poor field test. Furthermore, establishing the diagnosis library takes full advantage of the software's virtual computing capacity and the destruction which is taken by the physical injection is avoided.

\section{References}

[1] Ma Ya. Research on fault diagnosis of direct air-cooled condenser by simulation technology

[D].North China Electric Power University, 2013.

[2] Liao Guogang. Research on testability design of digital analog hybrid circuit [D]. China Academy of Engineering Physics, 2013.

[3] Shi Xianjun, Kong Dongming. Novel fault diagnosis approach of mixed-signal circuit [J].Ship Electronic Engineering, 2012, 01:83-84+102. 
[4] Xu Lei. Studies on Instantaneous Simulation Modeling of Domestic Electric Circuits by Cadence [J]. Journal of System Simulation, 2013, 12:2979-2985.

[5] Wang Jianhong, He Chuan, Wang Yi. Component modeling technology on simulation of complex analog circuits [J].Computer Engineering And Design, 2012, 12:4691-4695.

[6] Federico Bizzarri,Angelo Brambilla,Giancarlo Storti Gajani. Phase Noise Simulation in Analog Mixed Signal Circuits: An Application to Pulse Energy Oscillators.[J]. IEEE Trans. on Circuits and Systems,2011,58-II:. 